\title{
Measuring the gluon spin contribution to the proton spin in polarized $p+p$ collisions with the PHENIX experiment
}

\author{
Itaru Nakagawa ${ }^{1}$ for the PHENIX Collaboration \\ RIKEN \\ 2-1 Hirosawa, Wako, Saitama 351-0198 \\ E-mail: itarueriken.jp
}

\begin{abstract}
The gluon helicity distribution function $\Delta g(x)$ of the proton has been recognized as a fundamental quantity characterizing the inner structure of the nucleon. The Relativistic Heavy Ion Collider at Brookhaven National Laboratory provides unique opportunity to measure $\Delta g(x)$ using the polarized proton-proton collision at high energy. The double helicity asymmetry $A_{\mathrm{LL}}\left(p p \rightarrow \pi^{0} X\right)$ is sensitive to $\Delta g(x)$ and the golden probe for the PHENIX experiment owing to its abundant statistical samples. The global fit analysis result exhibited non-vanishing and positive $\Delta g(x)$ within its error by inclusion of the latest (Run9) $A_{\mathrm{LL}}$ for $\pi^{0}$ from PHENIX and jet from STAR measured at $\sqrt{s}=200 \mathrm{GeV}$. In this report, details of various $A_{\mathrm{LL}}$ measurements from Run 9 data samples are presented and their interpretation is to be discussed.
\end{abstract}

XXII. International Workshop on Deep-Inelastic Scattering and Related Subjects 28 April - 2 May 2014

Warsaw, Poland

\footnotetext{
${ }^{1}$ Speaker
} 


\section{Introduction}

Ever since the European Muon Collaboration (EMC) found that a surprisingly small fraction of the proton spin is carried by quark spins, substantial efforts are made both experimentally and theoretically in order to answer the fundamental question of QCD; "where does the rest of the proton spin come from?". Over the past 20 years, a large number of polarized lepton-nucleon deep inelastic scattering (DIS) experiments have established that only about $25-35 \%$ of the proton spin is carried by the quarks and antiquarks[1]. From the conservation principle of angular momentum, the rest of the spin must come from the intrinsic spin of the gluons $\Delta g(x)$ and the orbital angular momentum of quarks $L_{\mathrm{q}}$, and gluons $L_{\mathrm{g}}$. The polarized proton-proton collision at high energy in the Relativistic Heavy Ion Collider (RHIC) at BNL provides unique opportunity to access the gluon spin contribution to the proton spin. Several processes in particular jet or hadron production at high transverse momentum $p_{\mathrm{T}}$, receive substantial contribution from gluon-induced hard scattering in the leading order subprocess. Observed asymmetries of these production in polarized proton-proton collision thus have sensitivity to $\Delta g(x)$.

\section{The polarized proton-proton Collider and the PHENIX experiment}

RHIC has two rings, each containing up to 120 bunches of polarized protons (though usually not all "bunches" are filled). In the interaction regions, a bunch from one ring collides with a bunch from the other ring, then these same bunches circulate in opposite directions $3833 \mathrm{~m}$ around the RHIC rings and collide again about $13 \mu \mathrm{s}$ later. To preserve the spin during acceleration and store, a pair of Siverian snakes are used in

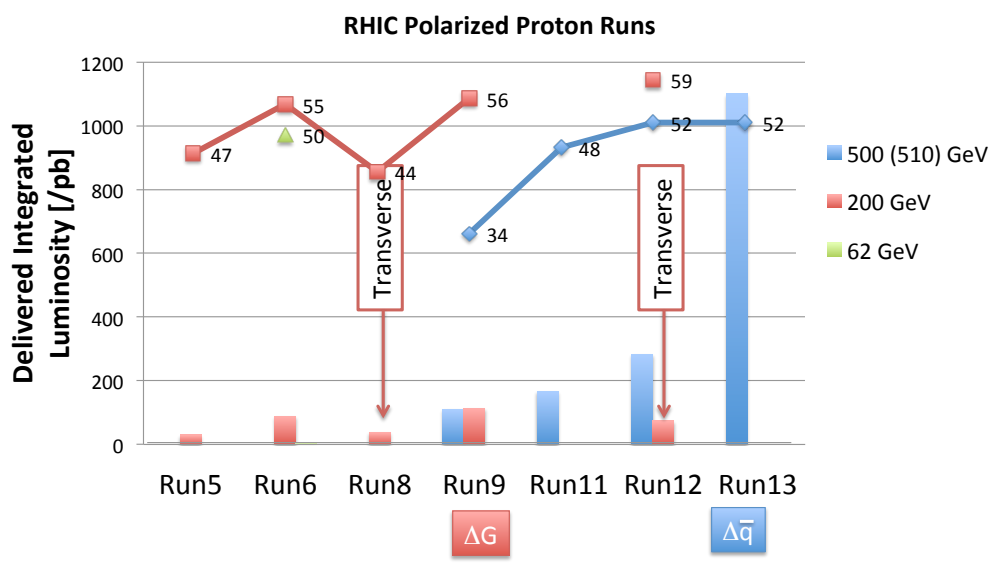

RHIC. Spin rotators can be used to rotate the spins from the stable vertical direction to the radial or longitudinal directions at the interaction region. The average polarization has been improved year by year as well as the luminosity, which allows us to obtain more integrated luminosity in shorter running time.

Figure 1 The delivered integrated luminosity and the average polarization of polarized proton beams in polarized proton beam experiments from 2005 (Run5) to 2013 (Run13).

Charted in Figure 1 is the delivered integrated luminosity to PHENIX and the average polarization for the polarized proton-proton experiments at RHIC from 2005 (Run5) to 2013 (Run13). The RHIC spin program has been focused on $\sqrt{s}=200 \mathrm{GeV}$ from Run5 to Run9 to measure $\Delta g(x)$ with various probes and then the focus has been taken 
over by the $\sqrt{s}=500 \mathrm{GeV}$ operation which highlights on the sea quark polarization measurement via W-boson production. The largest luminosity sample from Run9 at $\sqrt{s}=200 \mathrm{GeV}$ is the main focus of this report.

The PHENIX detector is designed to handle a high rate capability with limited acceptance with specialized triggers, high granularity detectors, and good mass resolution and particle identification. $\pi^{0}$ and $\eta$ mesons are detected by the finely grained central arm electromagnetic calorimeter (EMCal) covers the psudo-rapidity range of $|\eta|<0.35$ and forward muon piston calorimeter (MPC) $3.1<|\eta|<3.8$ (3.9).

\section{Double Helicity Asymmetry Measurements at RHIC}

PHENIX is sensitive to $\Delta g(x)$ through measurements of the double helicity asymmetries in the production of particular final state, such as $\pi^{0}$. The asymmetries are defined:

$$
A_{L L}^{\pi^{0}}=\frac{\sigma_{++} / d p_{T}-\sigma_{+-} / d p_{T}}{\sigma_{++} / d p_{T}+\sigma_{+-} / d p_{T}}
$$

where $\sigma_{++}\left(\sigma_{+-}\right)$is the production cross section for $\pi^{0}$ from $\mathrm{p}+\mathrm{p}$ collision with like (unlike) proton helicities. In leading order this can be factorized as the sum of all partonic subprocesses $a b \rightarrow c X$ where parton $c$ fragments into the detected $\pi^{0}$. In this frame work, $A_{L L}$ can be understood as the convolution:

$$
A_{L L}^{\pi^{0}}=\frac{\sum_{a b c} \Delta f_{a} \Delta f_{b} \hat{\sigma}(a b \rightarrow c X) \hat{a}_{L L}(a b \rightarrow c X) D_{c}^{\pi}}{\sum_{a b c} f_{a} f_{b} \hat{\sigma}(a b \rightarrow c X) D_{c}^{\pi^{0}}}
$$

where $f_{a}\left(\Delta f_{a}\right)$ are the unpolarized (polarized) parton distribution functions, and $D_{c}^{\pi}$ is the fragmentation function of parton $c$ into $\pi^{0}$. The spin-averaged partonic scattering cross-section for $a b \rightarrow c X$ is denoted by $\hat{\sigma}$ and $\hat{a}_{L L}$ is the analyzing power; both of which are calculable at next-to-leading order (NLO) in perturbative QCD (pQCD).

The $A_{L L}\left(p p \rightarrow \pi^{0} X\right)$ measurements have been the most sensitive probes of $\Delta g(x)$ in PHENIX thanks to their statistical abundance. $\pi^{0}$ is triggered by EMCal in the central arms through the decay mode $\pi^{0} \rightarrow \gamma \gamma$ with high efficiency. At $\sqrt{s}=200$ $\mathrm{GeV}$, the cross section is dominated by $q g$ scattering for $p_{T}>5 \mathrm{GeV}$ as shown in Figure 2 , so the process is directly sensitive to $\Delta g(x)$. 


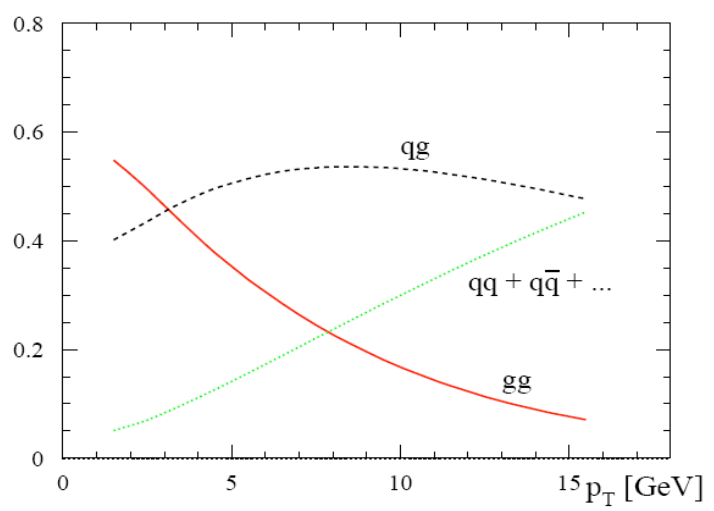

Figure 2 Decomposition of the NLO cross sections for $\mathrm{pp} \rightarrow \pi^{0} \mathrm{X}$ collisions into the contributions from initial $\mathrm{gg}, \mathrm{qg}$, and qq states [2].
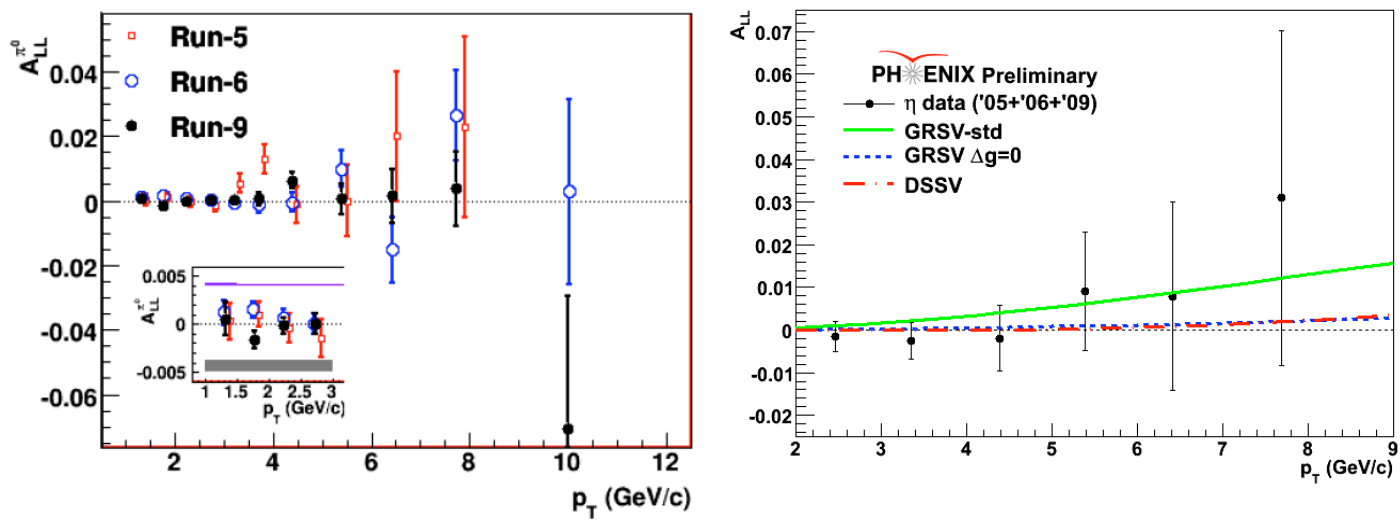

Figure 3 Measured $A_{L L}$ for $\pi^{0}$ (left) and $\eta$ (right) at $\sqrt{s}=200$ GeV in central rapidity region plotted as a function of $P_{T}$.

In the analyses, the invariant mass spectrum is formed from all pairs of photons, and the asymmetry is formed around the $\pi^{0}$ mass peak in a window from $112-162 \mathrm{MeV} / \mathrm{c}^{2}$. The background fraction and asymmetry in the background are estimated from side band around the mass peak. The background fraction varies from $49 \%$ in the $p_{\mathrm{T}}$ range of $1-1.5 \mathrm{GeV} / \mathrm{c}$, to $5.5 \%$ in the $12-15 \mathrm{GeV} / \mathrm{c}$ in the case of Run9 data. The analyses of $\eta$ are similar to these of $\pi^{0}$, but higher background contamination under the $\eta$ invariant mass peak. The $A_{L L}^{\eta}$ measurement has a slightly different sensitivity to $\Delta g(x)$ than $\pi^{0}$ since the flavor content of the $\eta$ is different. Shown in is the observed $A_{\mathrm{LL}}$ for $\pi^{0}$ (left) and $\eta$ (right) in the central rapidity region plotted as a function of $p_{\mathrm{T}}$. As can be seen from the comparison of Run5, 6 and $9 A_{L L}^{\pi^{0}}$ data[3], the statistical precisions are improved as Run goes by, resulting in very tight constraint on the extraction of $\Delta g(x)$ from data especially for Run9 ones. However the total uncertainty is limited by the systematic uncertainty (shaded band) in low $p_{\mathrm{T}}$ region, which is almost comparable to the statistical error of Run $9 \pi^{0}$ data. The dominant source of the systematic uncertainty comes from the relative luminosity between positive and negative bunches. 
These data are analyzed by the pQCD analysis framework [7] to extract $\Delta g(x)$ from observed asymmetries by the global fits to the available data sets including the latest inclusive Run9 jet $A_{\mathrm{LL}}$ data from STAR [4] and inclusive [5] and semi-inclusive [6] DIS data sets from COMPASS. As far as the impact on $\Delta g(x)$ is concerned, the data sets of Run9 PHENIX $\pi^{0}$ and STAR jet clearly dominate. Shown in Figure 4 is the global fit result[7] of $\Delta g(x)$ with (solid) and without (dot-dash) the latest (Run9) RHIC data sets. The solid curve exhibits the first non-vanishing positive result of the integral $\int_{0.05}^{0.2} d x \Delta g(x)=0.1 \pm_{0.07}^{0.06}$ for the $x$ range of $0.05 \leq x \leq 0.2$ spanned by the RHIC data at $Q^{2}=10 \mathrm{GeV}^{2}$ within in the error. The positive $\Delta g(x)$ is actually driven by the jet $A_{\mathrm{LL}}$ data from STAR [4] which covers higher $p_{\mathrm{T}}$ range $5 \lesssim p_{\mathrm{T}} \lesssim 30 \mathrm{GeV} / \mathrm{c}$ than $\pi^{0}$ data at PHENIX. However, the positive $\Delta g(x)$ fit is still consistent with PHENIX $\pi^{0}$ data within the error band as shown in right panel of Figure 4.
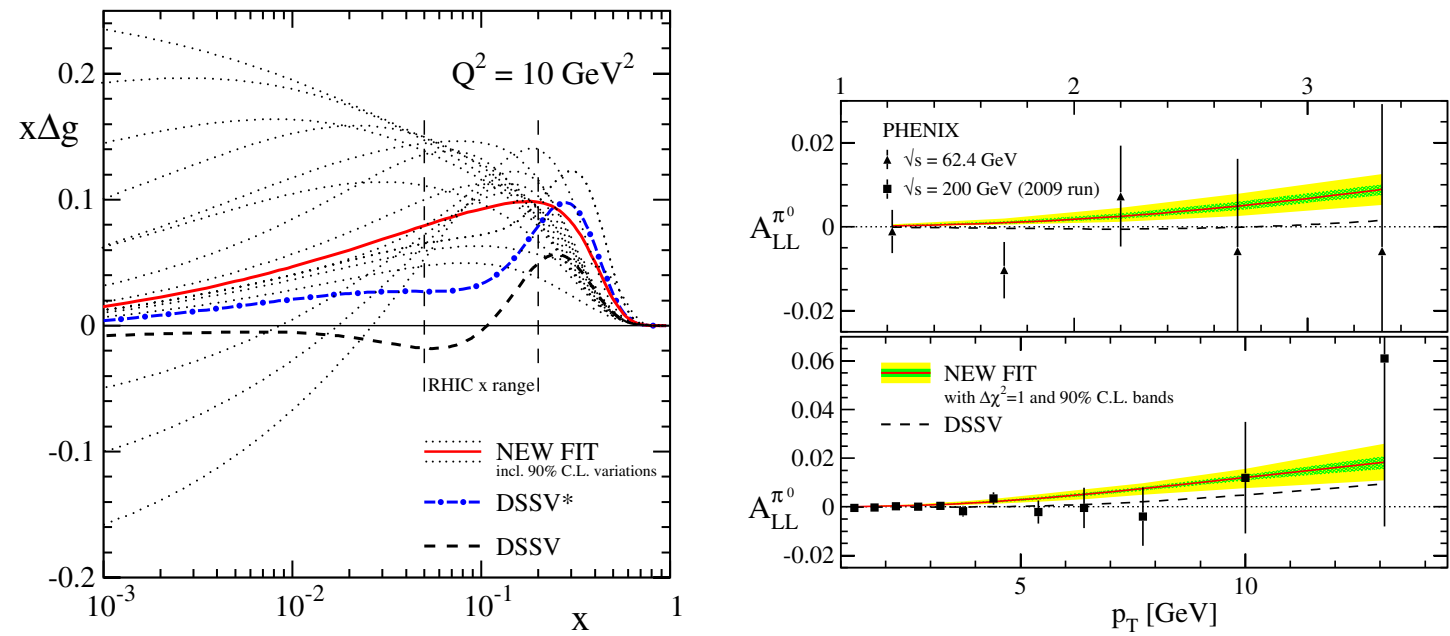

Figure 4 (left) Gluon helicity distribution at $Q^{2}=10 \mathrm{GeV}^{2}$ for the new fit including all the latest data sets, the original DSSV analysis of [8] and for an updated analysis without using the new 2009 RHIC data sets. The dotted lines present the gluon densities for alternative fits that are within the 90\% C.L. limit. The $x$ range primarily probed by the RHIC data is indicated by the two vertical dashed lines. (Right) PHENIX $\pi^{0}$ at $\sqrt{s}=62.4$ GeV and Rung $\sqrt{s}=200$ GeV data are compared with the DSSV fitting results. The inner and outer bands correspond to $\Delta \chi^{2}=1$ unit and $90 \%$ C.L., respectively.

On the other hand, efforts are made to extend the measured region of $\Delta g(x)$ to lower $x$ as low as $\sim 10^{-3}$ where gluon density gets larger using the forward muon piston detectors (MPCs). The detectors locate $2.20 \mathrm{~m}$ away from the collision vertex and therefore photon merging effects become significant for $E>20 \mathrm{G}$ photon(s) observed b $\mathrm{eV}$ or $p_{\mathrm{T}}>2 \mathrm{GeV} / \mathrm{c}$. For the analyses, the origin of the particle for the final state $\mathrm{y}$ the MPC is not identified, instead treated as single clusters, which are clusters consisted of electro-magnetic radiation in the calorimeters. The decomposition of original particle is thoroughly estimated by Monte Carlo full detector simulation. As shown in left panel of Figure 5 , the fraction of single cluster is dominated by $\pi^{0}$, but the fraction from other sources like direct photon and others moderately increase as a function of $p_{\mathrm{T}}$. The 
observed cluster $A_{\mathrm{LL}}$ at $\sqrt{s}=200 \mathrm{GeV}$ are plotted in the right panel of Figure 5. The data points exhibit consistent with zero asymmetry. The statistical errors are equally as small as Run9 central arm $\pi^{0}$, and the systematic error again compatible with the size of statistical error.
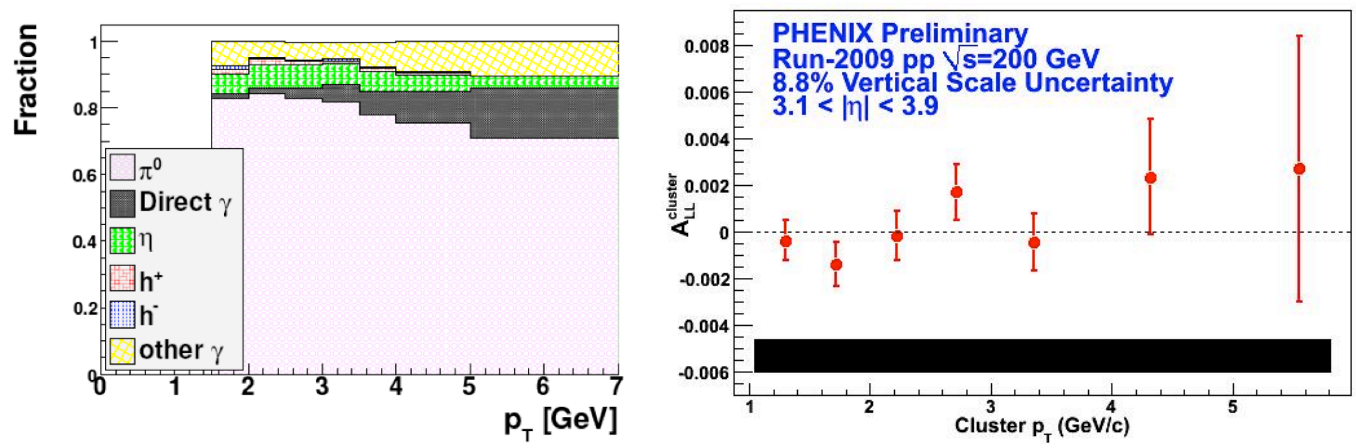

Figure 5 Run 9 Cluster $A_{L L}$ measured in forward rapidity region at $\sqrt{s}=200$ GeV. Shaded band represents the size of systematic uncertainly.

\section{Summary and Outlook}

We have measured polarized gluon distribution with various probes via double helicity asymmetry $A_{\mathrm{LL}}$ at $\sqrt{s}=200 \mathrm{GeV}$ in PHENIX. The result of the global fit analysis to the latest (Run 9) PHENIX $\pi^{0}$ in the central rapidity and STAR jet $A_{\mathrm{LL}}$ data exhibited non-vanishing and positive integrated gluon polarization function within the error in the region of $0.05<x<0.2$. It is important to extend the measurement of $\Delta g(x)$ to lower $x$ region where gluon density increases. New data from forward cluster and measurement at $\sqrt{s}=500 \mathrm{GeV}$ will explore unmeasured low $x$ region.

\section{References}

[1] For a review, see, e.g., C. A. Aidala, S. D. Bass, D. Hasch, and G. K. Mallot, Rev. Mod. Phys. 85, 655 (2013).

[2] V. Guzey, M. Strikman and W. Vogelsang, Phys. Lett. B 603, 173 (2004).

[3] A. Adare et al. (PHENIX Collaboration), arXiv:1402.6296.

[4] L.Adamczyketal.(STARCollaboration),arXiv:1405.5134.

[5] M.G. Alekseev et al. (COMPASS Collaboration), Phys. Lett. B 690, 466 (2010).

[6] M.G. Alekseev et al. (COMPASS Collaboration), Phys. Lett. B 693, 227 (2010).

[7] Daniel de Florian, Rodolfo Sassot, Marco Stratman, and Werner Vogelsang, Phys. Rev. Lett. 113, 012001 (2014).

[8] D. de Florian, R. Sassot, M. Stratmann, and W. Vogelsang, Phys. Rev. Lett. 101, 072001 (2008); Phys. Rev. D 80, 034030 (2009).. 